\title{
EVALUATION OF ANTIBACTERIAL ACTIVITY OF BIOSYNTHESIZED Ag-Au NANOCOMPOSITE USING VITIS VINIFERA FRUIT EXTRACT
}

\author{
Basavaraj Hiremath ${ }^{1, \bowtie}$ \\ ${ }^{1}$ Department of Chemistry, S. S. Margol Degree College, Shahabad-585 228, \\ Kalaburagi, Karnataka, India, \\ ${ }^{\otimes}$ Corresponding Author: drbhiremath25@gmail.com
}

\begin{abstract}
This report is an environmentally friendly biosynthesis method for the synthesis of Ag-Au bimetallic nanoparticles (Ag-Au NCP) from the equimolar solution of silver (I) nitrate and gold (III) chloride using Vitis vinifera fruit extract as a "green" reducing agent. This is a single-pot procedure and it doesn't involve any surfactant, template, and capping agent towards stabilization of nanoparticles. The crystallinity and morphology of the synthesized Ag-Au bimetallic nanocomposites were characterized using SEM, TEM, EDX, XRD, and FTIR. Additionally, the evaluation of antibacterial activity has been studied.
\end{abstract}

Keywords: Biosynthesis, Vitis vinifera, Antibacterial Activity, Bimetallic Ag-Au nanoparticles, Fruit Extracts.

RASĀYANJ. Chem., Vol. 14, No.4, 2021

\section{INTRODUCTION}

The biological production of nanoparticles is an area of great attention owing to its being less expensive and in the end it does not generate any hazardous chemicals during the process. ${ }^{1}$ In this process, the preparation of nanoparticles was performed by using microorganisms, plants, and their extracts. ${ }^{2}$ Though, microorganisms need a contaminated free culture medium to grow. ${ }^{3}$ Alternatively, plants and their extracts are abundant and easily accessible. Therefore, plants and their extract were deliberated more shows potential in the synthesis of nanoparticles in contrast with microorganisms and other testified processes. ${ }^{4}$ Plant extracts contain biological molecules with complex molecular structures, for example, amino acids, polysaccharides, vitamins, alkaloids, phenolics, terpenoids, saponins, and proteins, in rich. ${ }^{5}$ These plants derived bio-molecules act as reducing agents and further can be utilized in-situ reduction of metal salts into metallic nanoparticles lacking the adding up of external reducing agent. ${ }^{6}$ Additionally, these bio-molecules also afford stabilization to the nanoparticles and avoid the usage of expensive external capping agents. Silver (Ag) nanoparticles have developed as anti-microbial agents against multidrug-resistant bacteria and many industrial, pharmaceutical, biomedical, and environmental applications for the reason of their absolute physical and chemical features and high surface to volume ratio. $^{7}$ Accordingly, many types of research were executed for the preparation of $\operatorname{Ag}(0)$ nanoparticles using plant extracts, including their antioxidant, antifungal, and antibacterial activity assessments. ${ }^{8}$

Bimetallic nanoparticles research is one of the main significant zones in modern materials science studies. These nanoparticles show distinguished activities (e.g., catalytic, optical, cancer therapeutic and antimicrobial properties) in comparison to monometallic nanoparticles. ${ }^{9}$ That's why bimetallic nanoparticles were broadly developed in biomedical, catalysis, sensing, and electronics applications. Additionally, bimetallic nanoparticles have multi-functionalities such as 1) synergistic effect owing to the presence of dual metals; 2) an elevated number of effectual spots for the reason of their increased electromagnetic field on bimetallic interfaces. Several bimetallic nanoparticles, such as Ag-Pd, Ag-Ni, and $\mathrm{Ag}-\mathrm{Au}$, etc. were studied based on their different arrangements and morphologies. ${ }^{10}$ However, among various investigated bimetallic nanoparticles, $\mathrm{Ag}-\mathrm{Au}$ nanoparticles have got much consideration because of the high unswerving and pharmacological activities of gold (Au) nanoparticles and the abovementioned applications of silver nanoparticles. ${ }^{11}$

Rasayan J. Chem., 14(4), 2705-2710(2021)

http://dx.doi.org/10.31788/RJC.2021.1446467

This work is licensed under a CC BY 4.0 license. 
Previously numerous chemical and biological techniques were used for the synthesis of bimetallic nanocomposites. ${ }^{11}$ So far, no study has reported on "green synthesis" of silver-gold bimetallic nanoparticles by the use Vitis vinifera fruit extracts. In continuous of our research thrust toward a green and sustainable synthesis of nanomaterials, herein, we demonstrate a simple, economical, and ecofriendly technique for the synthesis of $\mathrm{Ag}$-Au bimetallic nanoparticles in using the Vitis vinifera fruit extracts along with their biological activity. The naturally occurring bio-molecules present in fruit extracts play a vibrant role in the preparation of nanocomposites without the addition of external reducing as well as capping agents.

\section{Materials and Methods}

\section{EXPERIMENTAL}

The reactants for analysis were crowdsourced from Merck (Mumbai, India). Deionized water is utilized for the preparation of Vitis vinifera fruit extract, $\mathrm{AgNO}_{3}$ (silver nitrate), $\mathrm{AuCl}_{3}$ (gold chloride) solutions. Glassware used was adequately flushed using chromic acid, and further, which is rinsed with deionized water and dehydrated. For the preparation of nanocomposite, silver nitrate, and gold chloride, ecological reduction by leaf extract is conducted at ambient temperature. The Agar disk diffusion procedure is used to assess the in vitro antimicrobial activity of the extract. ${ }^{12}$

\section{Preparation of Fruit Extract}

Vitis vinifera fruit is cleaned in deionized water and is left at room temperature to get dried, and the extract for the experiment is prepared from this fruit as exposed in Fig.-1. These fruits are sliced into bits and smashed in the grinder and filtered by using Whatman filter paper no.1. Using the filter paper with porousness lesser than the previously used to filter the obtained extract and is utilized as the Vitis vinifera fruit extract. ${ }^{13,14}$

\section{Synthesis of Ag-Au NCP}

An equal mixing of $1 \mathrm{mM}$ aqueous suspension of $\mathrm{AgNO}_{3}$ (silver nitrate) and $\mathrm{AuCl}_{3}$ (gold chloride) solutions formed in deionized water was utilized for formulating the Ag-Au salt solution. In a conical flask, $100 \mathrm{ml}$ of $1 \mathrm{mM} \mathrm{Ag-Au}$ salt solution and $10 \mathrm{ml}$ of Vitis vinifera fruit extract are added. Using a microwave oven with a frequency of $2.45 \mathrm{GHz}$ and power $800 \mathrm{~W}$, the prepared mixture of the solution is radiated with microwaves for around fifteen minutes and is kept in an appropriate sealing system at environment temperature for 24 hours. The biosynthesis reaction of Ag-Au nanoparticles depends on different parameters, like reaction time, metallic ion strength, the plant extract to metallic ions flow volume and the chemical reaction temperature. As a consequence of the reduction of Ag-Au salt solution, successive color changes from crystal clear to dark brown appeared, as exhibited in Fig.-2. Centrifugation of the sample extract was done for ten minutes at $500 \mathrm{rpm}$, succeeded by diffusion in $10 \mathrm{ml}$ deionized water. The alteration in the appearance of the color of the solution specifies the reduction of $\mathrm{Ag}^{+} \mathrm{into}_{\mathrm{Ag}^{0}}$ and $\mathrm{Au}^{++}$into $\mathrm{Au}^{0}$ and at the end, brown color signifies the production of $\mathrm{Ag}-\mathrm{Au} \mathrm{NCP}$. The final dark brown shade is a result of a reduction reaction. Ag-Au NCP formation and the entire reduction reaction happened only at a needed amount of fruit extract; hence minimal and optimal concentration of fruit extract is needful in the reaction method. ${ }^{15}$

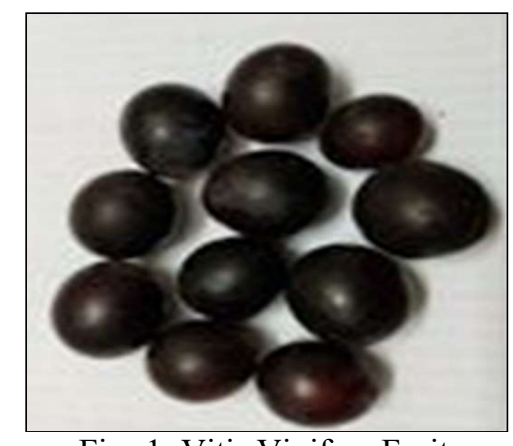

Fig.-1: Vitis Vinifera Fruit

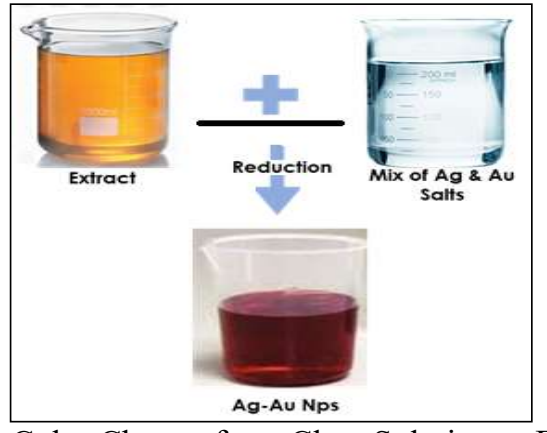

Fig.-2: Color Change from Clear Solution to Brown 


\section{Antibacterial Activity}

Ag-Au nanocomposite bactericidal activity was conducted in contrast with Bacillus subtilis (B. subtilis) and Klebsiella Planticola (K.Planticola) by utilizing the Agar disk diffusion technique. Using the LB broth method on a rotary shaker $(200 \mathrm{rpm})$ at about the temperature of $35^{\circ} \mathrm{C}$ and individual colonies of the sample strains were cultured at long night. Following the continuous incubation, the Muller-Hinton broth vehicle is employed to position a loop full of cultured bacteria. An aseptic disk is positioned superficial to the medium and is saturated with various concentrates of $10-50 \mu \mathrm{l}$ of Ag-Au NCP to assess the antibacterial properties of the composite particles. The zone of evolution suppression of the bacteria was evaluated after incubation at $35^{\circ} \mathrm{C}$ for two days. The silver ions hinder bacterial DNA reproduction by disrupting the sulfhydryl groups in protein and contacting the peptidoglycan cell wall and plasma membrane. The bactericidal action of Ag-Au NCP is subjective to the strength of nanoparticles pertain to the type of bacteria. ${ }^{16}$

\section{Characterization Study of synthesized Ag-Au NCP}

The morphological structure of the nanoparticles was determined by a Scanning Electron Microscope (SEM) by Philips (Amsterdam, The Netherlands). An X-ray diffractometer functioning at a voltage of 20 $\mathrm{kV}$ is utilized to identify the primary type of $\mathrm{Ag}$ and $\mathrm{Au}$ in the solution mixture. The Fourier transform infrared spectra (FTIR) of the samples data were recorded on a Perkin-Elmer FT-IR (Model No. 1000) in the range $4000-400 \mathrm{~cm}^{-1}$ at a resolution of $4 \mathrm{~cm}^{-1}$. A bright-field transmission electron microscopy image was obtained from JOEL $100 \mathrm{CX}$ operated at $190 \mathrm{KeV}$. JEOL JSM-6380 LA scanning electron microscope with energy dispersive microanalysis of X-Ray (EDAX) is used to study particle morphology with metal confirmation of the sample. ${ }^{17}$

\section{SEM Analysis}

\section{RESULTS AND DISCUSSION}

SEM analysis was conducted to study the morphology of the composite particles. SEM provides favorable evidence concerning the extent of agglomeration of particles, the size and its dissemination, shape, and surface morphology of the nanomaterials in its micro and nanoscales. The Ag-Au NCP characterization by the SEM is as represented in Fig.-3. Precise observation of the SEM image of NCP indicates the crystalline nature, the majority of the particles are relatively spherical-shaped, and obtained particles possess nano diameters. Some particles are in needle shape with a compact arrangement.

\section{TEM Analysis}

TEM technique has been utilized to understand the morphologies of the Ag-Au NCP. TEM is peculiar to discern and quantify the analytical dimensions, the electronic and chemical framework, and supreme spatially resolved characteristics of specific nanoparticles. The TEM image of the prepared Ag-Au NCP is shown in Fig.-4. TEM provides spherical and irregular-shaped particles of 100-500 nm size are shown in the images. Both $\mathrm{Ag}$ and $\mathrm{Au}$ particles are properly isolated by one other and obtained as crystalline nature, which was authenticated by the XRD diffraction data. Close compact structure and fine agglomeration can also be viewed in the image. Similar results were observed use of tea or coffee extract ranging from $5 \mathrm{~nm}$ to $100 \mathrm{~nm}$ size. TEM images pertain to Ag-Au nanocomposites were prepared from Cornelian cherry fruit extract as well illustrate pseudo spherical and spherical shapes.

\section{EDX Analysis}

The testing of the sample was employed by the EDX method to know the presence of silver and gold in silver-gold NCP's preparation. The EDX pattern exhibits the elemental composition and purity of the AgAu nanoparticles. The EDX pattern of the formed Ag-Au NCP is illustrated in Fig.-5. The EDX spectrum signifies the presence of silver and gold. The development of NCP is ascertained by distinct signals at the right kilo electron volt of $\mathrm{Ag}$ and $\mathrm{Au}$.

\section{X-ray Diffraction Analysis}

The crystalline phases of the nanoparticles are displayed using XRD analyses. X-ray diffraction analysis of the Ag-Au NCP is shown in Fig.-6. Consequently, the crystalline nature, the particles clearly show 
high intended Bragg's reflections. Au lattice planes reflections like (1 111$),\left(\begin{array}{lll}2 & 0 & 0\end{array}\right)$ and similarly Ag lattice planes $\left(\begin{array}{lll}1 & 0 & 0\end{array}\right),\left(\begin{array}{lll}2 & 2 & 0\end{array}\right),\left(\begin{array}{lll}3 & 1 & 1\end{array}\right)$ are registered. XRD pattern illustrates that the samples comprise Bragg's reflections of $\mathrm{Ag}$ as well as $\mathrm{Au}$ in composite form. The peaks noticed in the pattern indicate the crystallizing in the bio-organic phase on the face of the nanoparticles.

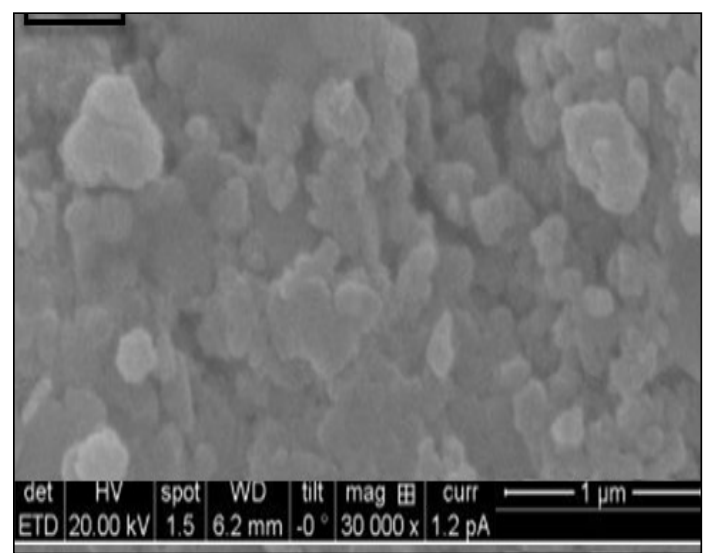

Fig.-3: SEM image of developed Ag-Au NCP

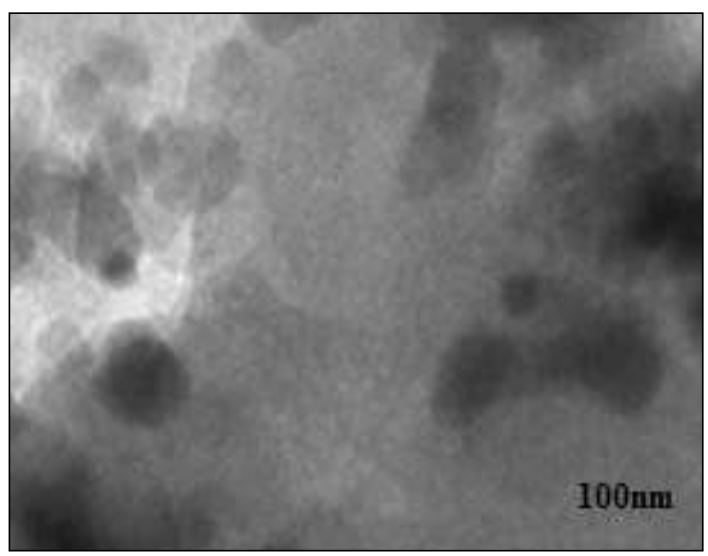

Fig.-4: TEM image of Ag-Au NCP

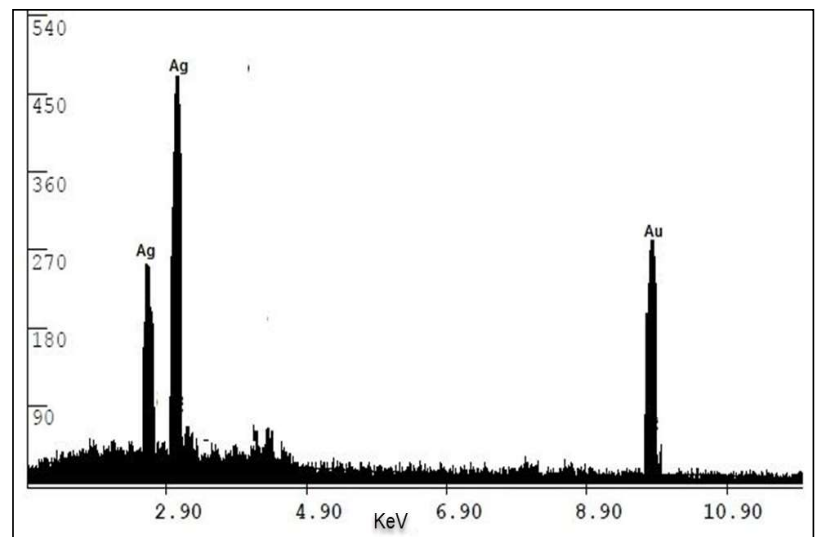

Fig.-5: EDX Pattern of Ag-Au NCP

\section{Fourier Transforms Infrared Spectroscopy}

FTIR spectroscopy is convenient for characterizing surface chemistry. Table-1 shows FT-IR data of Ag$\mathrm{Au}$ NCP obtained by fruit extract. The spectra show prominent absorption peaks at 3100, 1756, 1555, 1256, and $762 \mathrm{~cm}^{-1}$, respectively. The $\mathrm{N}-\mathrm{H}$ and $\mathrm{O}-\mathrm{H}$ extended oscillations of alcohol, amides, amines, and $\mathrm{H}$-bonded phenols pertain with the band range of $3100 \mathrm{~cm}^{-1}$, and the alkene $\mathrm{C}-\mathrm{H}$ oscillations are 
RASĀYAN J.Chem.

Vol. 14 | No. 4 |2705-2710| October- December | 2021

represented at the peak of $1756 \mathrm{~cm}^{-1}$. The $\mathrm{C}-\mathrm{H}$ in-plane bending of ester, ether, alkene, alcohol, and carboxylic acid presents at the peak of $1256 \mathrm{~cm}^{-1}$.

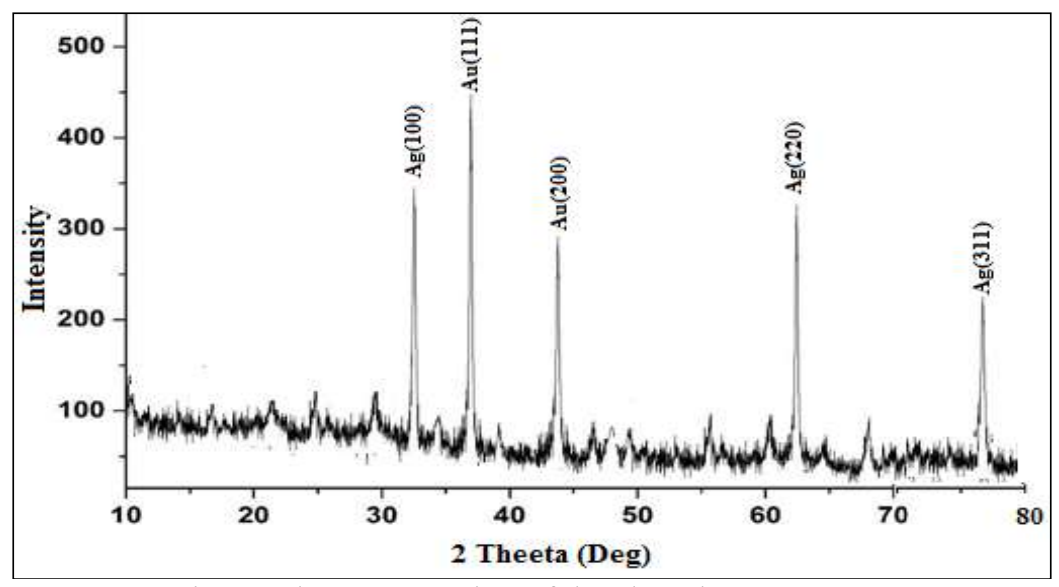

Fig.-6: The XRD Design of developed Ag-Au NCP

Table-1: Vibrational Bands of Ag-Au NCP Sample

\section{Antibacterial Activity}

\begin{tabular}{c|c}
\hline Sample & $\mathrm{Ag}-\mathrm{Au} \mathrm{NCP}$ \\
\hline Obtained Frequencies & $3100,1756,1555,1256,762$ \\
\hline
\end{tabular}

The contact of the Ag-Au nanoparticles with bacteria is ascertained by the size and shape. The larger the size, the more significant will be the bactericidal action. The Ag-Au nanocomposite particle's germicidal work was performed towards Klebsiella planticola (K.Planticola) and Bacillus subtilis (B.subtilis). By adding 10, 20,30,40, and $50 \mu \mathrm{l}$ of Ag-Au NCP in five consequent ways, the quantitative analysis was conducted. The size of the area of inhibition was assessed and is outlined in Table- 2 . The table proves that the nanoparticles are appropriate for use in antibacterial drugs. The sample shows considerable activity towards bacteria. Sample shows approximately same for $50 \mu 1$ sample. The clear observation table shows that the activity of the sample increases with an increase in the concentration of the sample. Furthermore, the smaller size Ag-Au NCP produced from the plant extract has the practical strength to enter the infective bacterial cells, destroying the proteins and exploding the cells, thus proving the antibacterial action. ${ }^{18}$

Table-2: Antibacterial Results of Ag-Au NCP (mm)

\begin{tabular}{c|c|c}
\hline \multirow{2}{*}{$\begin{array}{c}\text { The Concentration of Ag- } \\
\text { Au NCP }(\mu \mathrm{l})\end{array}$} & \multicolumn{2}{|c}{ Bacterial Strain (Zone of Inhibition) } \\
\cline { 2 - 3 } & B. Subtilis & K. Planticola \\
\hline 10 & 7 & 4 \\
\hline 20 & 7.5 & 5 \\
\hline 30 & 8.5 & 7 \\
\hline 40 & 10 & 9.5 \\
\hline 50 & 11 & 10.5 \\
\hline
\end{tabular}

CONCLUSION

We have effectively synthesized Ag-Au bimetallic nanoparticles through in-situ reduction of silver and gold salts using Vitis vinifera fruit extracts as a reducing agent and assessed them in antibacterial activity. Firstly, the structurally diverse biomolecules present in Vitis vinifera fruit extracts reduce the equimolar silver nitrate and gold chloride solution into Ag-Au bimetallic nanoparticles and then stabilize them without the addition of an external capping agent. This sustainable and green technology of Vitis vinifera fruit extracts can be industrialized for the large-scale production of other nanocomposites.

\section{ACKNOWLEDGEMENT}

The author thanks the Principal and staff members of S. S. Margol Degree College, Shahabad, for their constant support and encouragement. 
RASĀYAN J. Chem.

Vol. 14 | No. 4 |2705-2710| October- December | 2021

\section{REFERENCES}

1. G. Singhal, R. Bhavesh, K. Kasariya, A. R. Sharma and R. P. Singh, Journal of Nanoparticle Research, 13, 2981(2011), https://doi.org/10.1007/s11051-010-0193-y

2. M. N. Nadagouda, N. Iyanna, J. Lalley, C. Han, D. D. Dionysiou and R. S. Varma, ACS Sustainable Chemistry and Engineering, 2, 1717(2014), https://doi.org/10.1021/sc500237k

3. T. Riaz, P. Mughal, T. Shahzadi, S. Shahid and M. A. Abbasi, Materials Research Express, 6, 1250(2020), https://doi.org/10.1002/jsfa.2740610205

4. D. Hebbalalu, J. Lalley, M. N. Nadagouda and R. S. Varma, ACS Sustainable Chemistry and Engineering, 1, 703(2013),https://doi.org/10.1021/sc4000362

5. M. Cinelli, S. R. Coles, M. N. Nadagouda, J. Błaszczyński, R. Słowiński, R. S Varma and K. Kirwan, Green Chemistry, 17, 2825(2015), https://doi.org/10.1039/C4GC02088J

6. J. Singh, T. Dutta, K. H. Kim, M. Rawat, P. Samddar and P. Kumar, Journal of Nanobiotechnology, 16(1), 84(2018), https://doi.org/10.1186/s12951-018-0408-4

7. J. Lin, R. Chen, S. Feng, J. Pan, Y. Li, G. Chen, M. Chen, Z. Huang, Y. Yu and H. Zeng, Nanomedicine, 7(5), 655(2011), https://doi.org/10.1016/j.nano.2011.01.012

8. C. Krishnaraj, P. Muthukumaran, R. Ramachandran, M. D. Balakumaran and P. T. Kalaichelvan, Acalypha Indica Linn: Biotechnology Reports, 4, 42(2014), https://doi.org/10.1016/j.btre.2014.08.002

9. D. Wang and Yadong. Li, Advanced Materials, 23, 1044(2011), https://doi.org/10.1002/adma.201003695

10. L. Lei, R. Niu, and Y. Zhang, RSC advances, 8, 12428(2018), https://doi.org/10.1039/C8RA01343H

11. D. Wu and X. Liu, Applied Physics Letters, 97, 061904(2010), https://doi.org/10.1063/1.3479053

12. M. Balouini, M. Sadiki and S. K Ibnsouda, Journal of Pharmaceutical Analysis, 6(2), 71(2016), https://doi.org/10.1016/j.jpha.2015.11.005

13. A. Lagashetty, A. Pattar and S.K. Ganiger, International. Journal of Nano Dimension, 10(3), 291(2019), https://doi.org/10.1007/s00289-020-03403-0

14. A. Lagashetty, M. Patil and S. K. Ginger, Plasmonics, 8(3), 1219(2019), https://doi.org/10.1007/s11468-019-00910-3

15. G. Ghodake, S. R. Lim and D. S. Lee, Colloids and Surfaces B: Biointerfaces, 108, 147(2013), https://doi.org/10.1016/j.colsurfb.2013.02.044

16. M. P. Patil and G. D. Kim, Applied Microbiology and Biotechnology, 101(1), 79(2017), https://doi.org/10.1007/s00253-016-8012-8

17. A. Lagashetty, S. K. Ganiger and Shashidar, Heliyon, 5(12), e02794(2019), https://doi.org/10.1016/j.heliyon.2019.e027

18. M. G. Correa, F. B. Martínez, C. P. Vidal, C. Streitt, J. Escrig and C. L. Dicastillo, Beilstein Journal of Nanotechnology, 11, 1450(2020), https://doi.org/10.3762/bjnano.11.129

[RJC-6467/2021] 